\title{
LOCALLY FLAT STRINGS
}

\author{
BY CHARLES GREATHOUSE \\ Communicated by M. L. Curtis, February 4, 1964
}

I. The Schoenflies Theorem for strings. In [1], Stallings defines a string of type $(n, k)$ to be a pair $\left(R^{n}, Y\right)$, where $Y$ is a closed subset of $R^{n}$ such that $Y$ is homeomorphic to $R^{k}$. Similarly, he defines a pair $\left(S^{n}, X\right)$, where $X$ is homeomorphic to $S^{k}$, to be a knot of type $(n, k)$. A pair $(A, X)$ of $(n, k)$-manifolds is said to be locally smooth if each point of $X$ has a neighborhood $U$ in $A$ such that the pair $(U, U \cap X)$ is homeomorphic to the pair $\left(R^{n}, R^{k}\right)$. Thus, his definition of locally smooth is equivalent to Brown's [2] definition of locally flat.

Let $\left(R^{n}, Y\right)$ be a locally smooth string of type $(n, n-1) ; Y$ separates $R^{n}$ into two components whose closures are $A$ and $B$. In [1], Stallings states that it seems possible that either $A$ or $B$ must be homeomorphic to a closed half-space of $R^{n}$. Harrold and Moise [3] have proved this for $n=3$. In this note we observe that both $A$ and $B$ are closed half-spaces of $R^{n}$ for $n>3$ and hence we have a Schoenflies theorem for strings of type $(n, n-1)$ for $n>3$.

TheOREM I.1. Let $\left(R^{n}, Y\right)$ be a locally flat string of type $(n, n-1)$ and let $A$ and $B$ be the closures of the complementary domains of $Y$ in $R^{n}$. Then $A$ and $B$ are homeomorphic to a closed half-space of $R^{n}$ for $n>3$.

CoRollary I.2. Let $\left(R^{n}, Y\right)$ be a locally flat string of type $(n, n-1)$ for $n>3$. Then $\left(R^{n}, Y\right)$ is trivial, that is, there is a homeomorphism $h$ of $\left(R^{n}, Y\right)$ onto $\left(R^{n}, R^{n-1} \times 0\right)$.

Corollary I.3. Let $f_{1}, f_{2}$ be two locally flat embeddings of $R^{n-1}$ as a closed subset of $R^{n}$ for $n>3$. Then there is a homeomorphism $h$ of $R^{n}$ onto $R^{n}$ such that $h f_{1}=f_{2}$.

Theorem I.1 follows immediately from a recent result of Cantrell's [4]. Cantrell showed that a knot $\left(S^{n}, Y\right)$ of type $(n, n-1)$ is trivial for $n>3$ provided $Y$ is locally flat except at one point. Thus, if $\left(R^{n}, X\right)$ is a locally flat string of type $(n, n-1)$ and $\left(S^{n}, Y\right)$ is the one point compactification of $\left(R^{n}, X\right), Y$ is locally flat except at the compactification point. Hence $\left(S^{n}, Y\right)$ is trivial for $n>3$ and Theorem I.1 follows.

II. The Slab Conjecture. In this section we consider the relationship of locally flat strings of type $(n, n-1)$ to the Annulus Conjecture. We now state the Annulus Conjecture. 
II.1. The Annulus Conjecture. Let $S_{1}^{n-1}, S_{2}^{n-1}$ be two disjoint locally flat $n-1$ spheres embedded in $S^{n}$. Then the submanifold $M$ of $S^{n}$ bounded by $S_{1}^{n-1} \cup S_{2}^{n-1}$ is homeomorphic to $S^{n-1} \times[0,1]$.

Although the Annulus Conjecture is unsolved for $n>3$, the following theorem which is well known but does not seem to be in print holds.

THEOREM II.2. Let $S_{1}^{n-1}, S_{2}^{n-1}$ be two disjoint locally flat $n-1$ spheres embedded in $S^{n}$. Then if $M$ is the submanifold of $S^{n}$ bounded by $S_{1}^{n-1}$ $\cup S_{2}^{n-1}$ and $M_{i}=M-S_{i}^{n-1}, M_{i}$ is homeomorphic to $S^{n-1} \times[0,1)$ for $i=1,2$.

Proof. Let $A_{i}$ be the closed $n$-cell [2] with boundary $S_{1}^{n-1}$ such that $A_{i} \cap M=S_{i}^{n-1}$ for $i=1,2 . A_{i}$ is cellular and hence by Theorem I of [5], $S^{n} / A_{i}$ is homeomorphic to $S^{n}$ and the theorem follows.

A theorem analogous to Theorem II.2 holds for locally flat strings of type $(n, n-1)$ for $n>3$.

Theorem II.3. Let $R_{1}^{n-1}, R_{2}^{n-1}$ be two disjoint locally flat $n-1$ planes embedded as closed subsets of $R^{n}$ for $n>3$. Then if $M$ is the submanifold of $R^{n}$ bounded by $R_{1}^{n-1} \cup R_{2}^{n-1}$ and $M_{i}=M-R_{i}^{n-1}, M_{i}$ is homeomorphic to $R^{n-1} \times[0,1)$ for $i=1,2$.

Proof. In view of Corollary I.2, we may assume that $R_{1}^{n-1}=R^{n-1} \times 0$ and $R_{2}^{n-1} \subset R^{n-1} \times(0, \infty)$. Let $A_{2}$ be the closed half-space (by Theorem I.1) of $R^{n}$ bounded by $R_{2}^{n-1}$ which does not contain $R_{1}^{n-1}$. By Theorem I.1, $R^{n}-A_{2}$ is homeomorphic to $R^{n}$ and hence by the same theorem $M_{2}$ is homeomorphic to $R^{n-1} \times[0,1)$. Similarly, $M_{1}$ is homeomorphic to $R^{n-1} \times[0,1)$.

We now state the Slab Conjecture.

II.4. The Slab Conjecture. Let $R_{1}^{n-1}, R_{2}^{n-1}$ be disjoint locally flat $n-1$ planes embedded as closed subsets of $R^{n}$. Then if $M$ is the submanifold of $R^{n}$ bounded by $R_{1}^{n-1} \cup R_{2}^{n-1}, M$ is homeomorphic to $R^{n-1} \times[0,1]$.

It should be noted that the Slab Conjecture is false in dimension 3. A counterexample can be obtained as follows. Let $S_{1}^{2}$ be the 2 -sphere boundary of a 3-cell obtained by "swelling" a Fox-Artin arc (Example 1.2) [6]. We may assume that $S_{1}^{2}$ is contained in the unit 3-ball $B^{3}$ of $S^{3}$, that $S_{1}^{2} \cap \dot{B}^{3}=p$, and that $S_{1}^{2}$ is locally flat at each point other than $p$. Let $S_{2}^{2}=\dot{B}^{3}, R_{1}^{2}=S_{1}^{2}-p$ and $R_{2}^{2}=S_{2}^{2}-p$. Then $R_{1}^{2}, R_{2}^{2}$ are disjoint locally flat 2-planes embedded as closed subsets of $R^{3}=S^{3}-p$. The 3-dimensional Slab Conjecture would imply that the closure of the complementary domain of $S_{1}^{2}$ in $S^{3}$ containing $R_{2}^{2}$ is a closed 3-cell which is a contradiction since $S_{1}^{2}$ is wild in $S^{3}$. 
The Slab Conjecture is unsolved for $n>3$ and the following theorem indicates that it is possibly stronger than the Annulus Conjecture.

Theorem II.5. The Slab Conjecture implies the Annulus Conjecture for $n>3$.

PRoOF. Let $S_{1}^{n-1}, S_{2}^{n-1}$ be disjoint locally flat $n-1$ spheres embedded in $S^{n}$. In view of Brown's theorem [2], we may assume that $S_{1}^{n-1}=S^{n-1}=$ the equator of $S^{n}$ and $S_{2}^{n-1}$ lies in the northern hemisphere of $S^{n}$. Now there is a unique $n-1$ sphere $S_{\beta}^{n-1}$ with the following properties:

(1) $S_{\beta}^{n-1}$ lies in the northern hemisphere of $S^{n}$.

(2) $S_{\beta}^{n-1}$ is concentric with $S^{n-1}=$ the equator of $S^{n}$.

(3) $S_{\beta}^{n-1} \cap S_{2}^{n-1}$ is not empty.

(4) The half-open annulus bounded by $S^{n-1} \cup S_{\beta}^{n-1}$ but not containing $S_{\beta}^{n-1}$ does not intersect $S_{2}^{n-1}$.

Let $p \in S_{\beta}^{n-1} \cap S_{2}^{n-1}$ and let $D^{n-1}$ be the standard unit $n-1$ cell in $S^{n-1}$ with center $p^{\prime}$ where $p^{\prime}$ and $p$ lie on a great circle passing through the north pole. Let $C$ be the cone over the base $\dot{D}^{n-1}$ with vertex $p$. Then $\left[S_{1}^{n-1}-\operatorname{Int}\left(D^{n-1}\right)\right] \cup C=S_{3}^{n-1}$ is a locally flat $n-1$ sphere such that $S_{3}^{n-1} \cap S_{2}^{n-1}=p$.

If we define $R_{1}^{n-1}=S_{3}^{n-1}-p$ and $R_{2}^{n-1}=S_{2}^{n-1}-p$, then $R_{1}^{n-1}, R_{2}^{n-1}$ are disjoint locally flat $n-1$ planes embedded as closed subsets of $S^{n}-p$ $=R^{n}$. By the Slab Conjecture, the submanifold $N^{n}$ bounded by $R_{1}^{n-1} \cup R_{2}^{n-1}$ in $R^{n}$ is homeomorphic to $R^{n-1} \times[0,1]$. Hence, there is a homeomorphism $h$ of $N^{n}$ onto $R^{n-1} \times[0,1]$ where $h\left(R_{1}^{n-1}\right)=R^{n-1} \times 0$ and $h\left(R_{2}^{n-1}\right)=R^{n-1} \times 1$. Since $\dot{D}^{n-1}$ is a flat $n-2$ sphere in $R_{1}^{n-1}$, $h\left(\dot{D}^{n-1}\right)$ is a flat $n-2$ sphere in $R^{n-1} \times 0$. Therefore, there is a homeomorphism $g$ of $R^{n-1} \times 0$ onto itself such that $g h\left(\dot{D}^{n-1}\right)$ is the standard unit $n-2$ sphere $S_{1}^{n-2}$ in $R^{n-1} \times 0 . g$ extends naturally to a homeomorphism $G$ of $R^{n-1} \times[0,1]$ onto itself by $G(x, t)=(g(x), t)$. Then $k=G h$ is a homeomorphism of $N^{n}$ onto $R^{n-1} \times[0,1]$ such that $k\left(\dot{D}^{n-1}\right)$ is the standard unit $n-2$ sphere $S_{1}^{n-2}$ in $R^{n-1} \times 0$.

Consider an $n$-annulus $S^{n-1} \times[0,1]=A^{n}$. Let $q \in S^{n-1} \times 0$ and $B^{n-1}$ be the unit $n-1$ cell in $S^{n+1} \times 0$ with center $q$. Let $q^{\prime}=(q, 1) \in S^{n-1}$ $\times 1$ and $C^{\prime}$ be the cone with base $\dot{B}^{n-1}$ and vertex $q^{\prime}$. Take $F^{n}$ to be the $n$-cell in $A^{n}$ with boundary $C^{\prime} \cup B^{n-1}$ and let $L^{n}=A^{n}$ $-\left[\operatorname{Int}\left(F^{n}\right) \cup \operatorname{Int}\left(B^{n-1}\right) \cup q^{\prime}\right]$.

There is a homeomorphism $j$ of $R^{n-1} \times[0,1]$ onto $L^{n}$ such that $j\left(R^{n-1} \times 1\right)=\left(S^{n-1} \times 1\right)-q^{\prime}, j\left(R^{n-1} \times 0\right)=\left[\left(S^{n-1} \times 0\right)-\operatorname{Int}\left(B^{n-1}\right)\right]$ $\cup\left(C^{\prime}-q^{\prime}\right)$ and $j\left(S_{1}^{n-2}\right)=\dot{B}^{n-1}$. Then $f=j k$ is a homeomorphism of $N^{n}$ onto $L^{n}$ such that $f\left(\dot{D}^{n-1}\right)=\dot{B}^{n-1}$. $f$ extends uniquely to a homeomor- 
phism $F$ of $N^{n} \cup p$ onto $L^{n} \cup q^{\prime}$ by taking $F(p)=q^{\prime}$.

Finally, let $M$ be the submanifold of $S^{n}$ bounded by $S_{1}^{n-1} \cup S_{2}^{n-1}$. Since $F(C)=C^{\prime}, F$ extends to a homeomorphism of $M$ onto $A^{n}$ by extending first to take $D^{n-1}$ onto $B^{n-1}$ and finally extending to take the $n$-cell bounded by $C \cup D^{n-1}$ onto $F^{n}$. Thus, $M$ is homeomorphic to $S^{n-1} \times[0,1]$ and the theorem is proved.

It does not seem obvious that the Annulus Conjecture implies the Slab Conjecture for $n>3$.

\section{REFERENCES}

1. J. Stallings, On topologically unknotted spheres, Ann. of Math. (2) 77 (1963), 490-503.

2. M. Brown, Locally flat imbeddings of topological manifolds, Ann. of Math. (2) 75 (1962), 331-341.

3. O. G. Harrold, Jr. and E. E. Moise, Almost locally polyhedral spheres, Ann. of Math. (2) 57 (1953), 575-578.

4. J. C. Cantrell, Almost locally flat embeddings of $S^{n-1}$ in $S^{n}$, Bull. Amer. Math. Soc. 69 (1963), 716-718.

5. M. Brown, $A$ proof of the generalized Schoenflies theorem, Bull. Amer. Math. Soc. 66 (1960), 74-76.

6. E. Artin and R. H. Fox, Some wild cells and spheres in three-dimensional space, Ann. of Math. (2) 49 (1948), 979-990.

UNIVERSITY OF TENNESSEE 